\title{
Model Research of Database Dynamic Pricing Based on DDLG Danmin Qian ${ }^{1,2, a}$, Kui Jiang ${ }^{1, b}$, Yuanpeng Zhang ${ }^{1, \mathrm{c}}$, Min Yao ${ }^{3, \mathrm{~d}}$ and Li Wang ${ }^{1, \mathrm{e},{ }^{*}}$ \\ ${ }^{1}$ Department of Medical Informatics, Medical School, Nantong University, 19 Qixiu Road, Nantong 226001, Jiangsu Province, China \\ ${ }^{2}$ Information Management College, Nanjing University, China \\ ${ }^{3}$ Department of Immunology, Medical School, Nantong University, 19 Qixiu Road, Nantong 226001, Jiangsu Province, China \\ asandy8793@ntu.edu.cn, bkuij@ntu.edu.cn, cmaxbirdzhang@ntu.edu.cn, ${ }^{\mathrm{a}}$ erbei@ntu.edu.cn, e,*wangli@ntu.edu.cn (Corresponding author)
}

Key words: dynamic pricing; database; electronic resources

\begin{abstract}
Based on the main functional characteristics of ITM model, and combined the sharing, replication, fugitiveness, dynamic characteristics of database, created the DDLG model for efficient database .Use an example for the case simulation, perfectly verified the feasibility and effectiveness of this set of dynamic pricing model.

In a big wave of resources digitization, the database's status is becoming more and more important, but the price of database is hard to make correctly. The author carries on some related researches refer to dynamic pricing methods of economics.
\end{abstract}

\section{Introduction dynamic pricing}

The database dynamic pricing is formulating the right price based on the database inventory information's value, different consumer needs, and combined with the market situation at different times of the change trend [1].

The products in the database is the electronic resources, so it is different from any other products, it has special qualities such as it can be shared, be copied, the perishable, development costs a one-time, its pricing is more complex than the general products. If the price is too high, may make consumers away, but if the price is too low, even can't reach a certain sales, it may be at a loss. On the above features, author based on the research of traditional dynamic pricing methods of ITM, created the D (Direct marketing), D (Dynamic pricing), L(Large goods), G (Group) model.

\section{Build DDLG model}

DDLG model requires several assumptions:

Hypothesis 1: On the market, there are two companies has products with the similar function.

Hypothesis 2: Consider the long-term interests and competitors, companies develop products price level to achieve the benefit maximization.

Hypothesis 3: Enterprise sales cycle always for T.

Hypothesis 4: Customer for the product has no strategic behavior.

\subsection{Demand function}

Periodically sales, product cycle is $\mathrm{T}$, and each cycle pricing is $\mathrm{Pt}$; there is one of the biggest potential market demand is a; the actual demand for the corresponding cycle is $\mathrm{D}$; because the product pricing has influence to the market demand, the impact factors of reason set pricing for demand is b; then the demand function is: $D=a-b P_{t}$

Factors that affect market demand include the enterprise and competitors' product pricing and the quality of service [5]. This paper set the consumer of subjective value is $P_{i t}^{*}$, Consumers purchase decisions ultimately largely depends on the subjective value differences with the actual value of the product. Comprehensive above factors can be concluded that the products of this enterprise demand 
function is:

$D_{i t}=A_{i t}-b P_{i t}-g P_{i(i-1)}+\alpha S_{i t}+\beta S_{i(t-1)}+\lambda P_{j t}+h P_{j(t-1)}-\rho S_{j t}-\eta S_{j(t-1)}+l P_{i t}^{*}$

$b, g, \alpha, \beta, \lambda, h, \rho, \eta, l \in[0,1]$

In the function (2), $\mathrm{i}, \mathrm{j}$, respectively, represents the company and competitors, Ait is according to the demand of the enterprise period $t-1$, for the period $t$ basic demand of the market (Ait=Di(t-1)), When $t=1$, the product has just listed, there is no demand for any sales situation, Ai1 is equal to zero, Clearly the company's overall demand Di1 $>0$.

Pit and Pjt is the products pricing in period t respectively for enterprise and competitors. Dit increases with Pjt increases and Pit decreases. $\mathrm{b}$ and $\lambda$ are the sensitive coefficient for this enterprise and competitor product pricing compare to the demand of the product. Sit, Sjt is the quality of service of this company and competitors in the period t.

$\mathrm{Pi}(\mathrm{t}-1)$ and $\mathrm{Pj}(\mathrm{t}-1)$ is product pricing of this company and competitors in phase $\mathrm{t}-1 \mathrm{~g}$ and $\mathrm{h}$ are respectively the impact factor of $\mathrm{Pi}(\mathrm{t}-1)$ and $\mathrm{Pj}(\mathrm{t}-1)$ to $\mathrm{Dit}$. $\mathrm{Si}(\mathrm{t}-1)$ and $\mathrm{Sj}(\mathrm{t}-1)$ is the quality of service of this company and competitors in the period $\mathrm{t}-1 . \lambda$ and $\eta$ is impact factor of the $\mathrm{Si}(\mathrm{t}-1)$ and $\mathrm{Sj}(\mathrm{t}-1)$ relative to Dit [2], so $\Delta_{i t}=h P_{j(t-1)}-g P_{i(t-1)}+\beta S_{i(t-1)}-\eta S_{j(t-1)}$ is the product price and the service level of "learning" effect in phase $t-1$ for the enterprise's change of product demand in phase t.

$l$ is consumers' subjective factor of the influence of value to the product demand, consumers' subjective value to buy the product value of decision-making as the factor increases.

2.2 Service cost function

$$
\text { Assuming that the service cost function is : } \quad C_{i t\left(S_{i t}\right)}=\frac{k}{2}\left(S_{i t}\right)^{2}
$$

$k$ is impact factor of the enterprise providing the quality of service to the service cost. Enterprise service ability will influence the size of the factor k.2.3 Revenue function

$$
\pi_{i t}=\left(P_{i t}-C_{i}\right) D_{i t}-C_{i t\left(S_{i t}\right)}
$$

2.4 Model analysis

2.4.1 The optimal equilibrium price of products

Type (4) to $P_{i t}$ a derivative and second derivative, respectively, to:

$\frac{\partial \pi_{i t}}{\partial P_{i t}}=D_{i t}-b\left(P_{i t}-C_{i}\right)$

$$
\frac{\partial \pi_{i t}^{2}}{\partial P_{t i}^{2}}=-2 b
$$

By $b \in[0,1]$, available $\frac{\partial_{\pi_{t i}}^{2}}{\partial P_{i t}^{2}}<0$, so the existing enterprise optimal equilibrium price.

According to the formula (5), $\frac{\partial \pi_{i t}}{\partial P_{i t}}=0$ get the enterprise of optimal equilibrium price in phas
\[ P_{i t}=\frac{\left(A_{i t}+\alpha S_{i t}-\rho S_{j t}+\Delta_{i t}+1 P_{i t}^{*}-C_{i}\right)+\lambda\left(A_{j t}+\alpha S_{j t}-\rho S_{i t}+\Delta_{j t}+1 P_{j t}^{*}-C_{j}\right)}{2 b} \]

It finds that no matter what circumstances the optimal price of the product and the consumer of the product value valuations are negative correlation [3].

2.4.2 Consumer value evaluation of the impact analysis of the optimal equilibrium price products

Formula (7) to $P_{i t}^{*}$ derivation: $\frac{\partial P_{i t}}{\partial P_{i t}^{*}}=\frac{l}{2 b}$

From this we can get $\frac{\partial P_{i t}}{\partial P_{i t}^{*}}>0, P_{i t}$ is monotone increasing function on $P_{i t}^{*}$, products optimal equilibrium price and $P_{i t}^{*}$ is positive correlation.

2.4.3 Service quality impact analysis of the optimal equilibrium price products ${ }^{[4]}$

$S_{\text {it }}$ and $P_{i t}$ is positive correlation. 
Formula (7) to $S_{i t}$ derivation: $\frac{\partial P_{i t}}{\partial S_{i t}}=\frac{\alpha}{2 b}$

In the same way, $\frac{\partial P_{i t}}{\partial S_{j t}}=-\frac{\rho}{2 b}, \frac{\partial P_{i t}}{\partial S_{j t}}<0 . P_{i t}$ is negative with $S_{j t}$.

By the analysis above, we can get: $\frac{\partial P_{i t}}{\partial S_{i t}}>0, \frac{\partial P_{i t}}{\partial S_{j t}}<0$. The product demand to the service level of enterprises sensitivity is greater than the competitors, but when the price of the sensitive coefficient is less than the competitors, the optimal equilibrium price of products and the enterprise service level positively correlated, and negatively correlated with rival service quality.

\section{Case simulation}

3.1 Model of market demand and total revenue

This model is applicable to any database, however the sensitivity value of different size of database is different, according to the characteristics of the small and medium-sized database, author set up corresponding sensitive coefficient. Will an enterprise database of product data (such as Tabel 1) into the formula (2), according to the R language visualization function persp ().

This article set $\mathrm{b}=0.7, \mathrm{~g}=0.4, \mathrm{~h}=0.5, \beta=\eta=0.4, \lambda=0.6, \alpha=0.6, \rho=0.5, \quad l=0.3$.

The market demand function: $\quad \mathrm{D}_{\mathrm{it}}=123000-0.7 \mathrm{P}_{\mathrm{it}}+0.6 \mathrm{~S}_{\mathrm{it}}$

Tabel1 An enterprise database product data

\begin{tabular}{|c|c|c|}
\hline Index & Phase t-1 & Phase t \\
\hline $\operatorname{cost}_{C_{i}}$ (unit: yuan) & 40000 & 40000 \\
\hline Market demand $D_{i t}$ & 100000 & \\
\hline Product price $_{P_{i t}}$ (unit: yuan per year $)$ & 100000 & \\
\hline Competitor's product price $_{P_{j t}}$ （unit: yuan per year） & 98000 & 95000 \\
\hline Service quality $S_{i t}$ & 90000 & \\
\hline Competitor's service quality $P_{j t}$ & 85000 & 90000 \\
\hline Consumers subjective price of the enterprise database $P_{i t}^{*}$ (unit: yuan) & & 94000 \\
\hline Consumers subjective price of the competitor's database $P_{j t}^{*}$ (unit: yuan) & & 93000 \\
\hline
\end{tabular}

Then computing the enterprise service cost function in phase $\mathrm{t}: C_{i t\left(S_{i t}\right)}=\frac{k}{2}\left(S_{i t}\right)^{2}$, under the condition of known $\mathrm{k}$ value, the enterprise to provide service for the customer the service cost coefficient is 0.4, namely: $C_{i t\left(S_{i t}\right)}=0.2\left(S_{i t}\right)^{2}$

Thus we can get enterprise total revenue function expressions of the phase $t$ as follows:

$$
\pi_{i t}=\left(P_{i t}-C_{i}\right) D_{i t}-0.2\left(S_{i t}\right)^{2}
$$

That is $\pi_{i t}=\left(P_{i t}-40000\right)\left(51300-0.7 P_{i t}+0.6 S_{i t}\right)-0.2\left(S_{i t}\right)^{2}$

$\mathrm{R}$ language code of three-dimensional figure for the relationship between market demand, service quality and pricing is:

$$
\text { database price<-seq(40000,120000,10000) }
$$

service quality<-seq $(0,100000,10000)$

function $1<$-function(database price, service quality) $123000-0.7 *$ database price $+0.6 *$ service quality

market demand<-outer(database price, service quality, function1)

persp(database price, service quality, market demand, theta=30,phi=30,expand=0.7,col="green") 


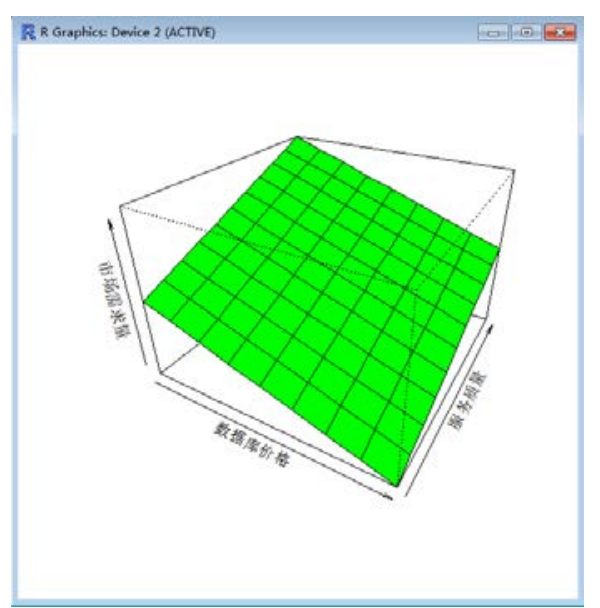

Fig1 relationship between market demand $\backslash p r i c e l s e r v i c e$ quality

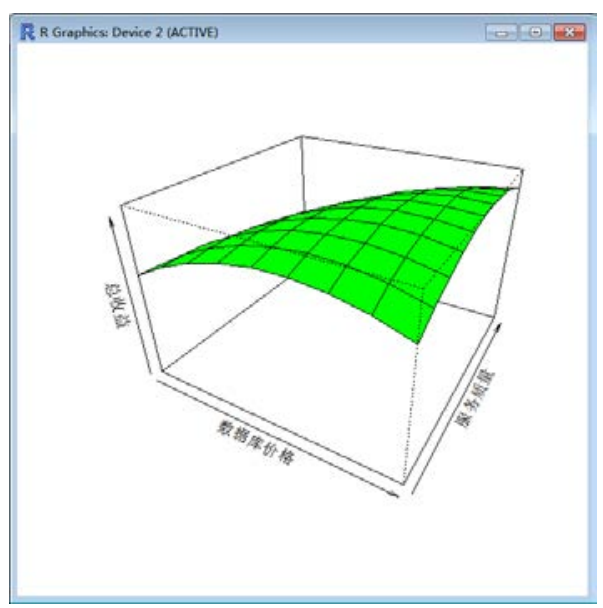

Fig2 relationship between total revenuelpricinglservice quality

By Fig 1, the market demand is affected by the pricing and service quality. When service quality remains, the market demand is decreased with the increase of price. When price remains, the market demand increases with the improvement of service quality. When price high and service quality low, the market demand is the lowest.

$\mathrm{R}$ language code of three-dimensional figure for the relationship between total revenue, service quality and pricing is:

database price<-seq(40000,120000,10000)

service quality<-seq $(0,100000,10000)$

function $2<$-function(database price, service quality)(database price-40000)*(123000-0.7*database price $+0.6 *$ service quality) $-0.4 *(\text { service quality })^{\wedge} 2$

total revenue<-outer(database price, service quality, function 2)

persp(database price, service quality, total revenue, theta=30,phi=30,expand=0.7,col="green")

By Fig 2, the total revenue is influenced by pricing and service quality. When the service quality is constant, within a certain range, the total revenue increased with the increase of pricing; more than the range, then decreased with the increase of price. When the price is changeless, within a certain range, the total revenue increases with the improvement of service quality; Exceeds the range, the services provided by the cost of enterprises has greatly increased, so with the improvement of service quality and reduce the total revenue. Pricing would be a reasonable price, appropriate to improve the quality of service, can make the total return to obtain maximum.

\subsection{Model establishment and analysis of the price and quality}

In order to improve service quality for higher optimal equilibrium price, we have carried on related research activities to observe the relationship between them. We stimulated the obtained data, made the enterprise and the competition of quality of service in the corresponding reasonable scope.

\subsubsection{Relationship between low levels of service quality and the optimal equilibrium price}

Experiment assumption competitor's service quality is 10, the company's service quality control in the range of 1 to 10 , and the corresponding data into $P_{i t}^{*}$ expression is obtained:

$$
\mathrm{P}_{\mathrm{it}}=0.43 \mathrm{~S}_{\mathrm{it}}+111996
$$

We assuming $S_{\text {it }}$ here for $x$, R language mapping (as shown in Fig 3 ) and related $R$ language code is as follows ${ }^{[5]}$ : curve $\left(0.43^{*} x+111996,0,10\right)$

3.2.2 Relationship between medium level of service quality and the optimal equilibrium price

Experiment will assume that rival service quality for 1000, and the quality control of this enterprise in the range of 1-1000, and the corresponding data into this enterprise in the expression of the optimal equilibrium price $P_{i t}^{*}$ is obtained: $\mathrm{Pit}=0.43 \mathrm{Sit}+111643$ 
Related R language code as follows, generated as shown in Fig 4:

curve $(0.43 * x+111643,0,1000)$

3.2.3 Relationship between higher level of service quality and the optimal equilibrium price

Experiment will assume that rival service quality for 100000 , and the quality control of this enterprise in the range of 1-100000, and the corresponding data into this enterprise in the expression of the optimal equilibrium price $P_{i t}^{*}$ is obtained: $\mathrm{Pit}=0.43 \mathrm{Sit}+76286$

Related R language code as follows, generated as shown in Fig 5:

curve $(0.43 * x+76286,0,100000)$

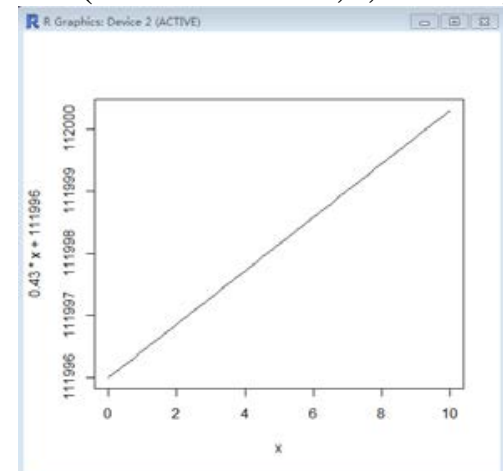

Fig3 Relationship between low levels of service quality and the optimal equilibrium price

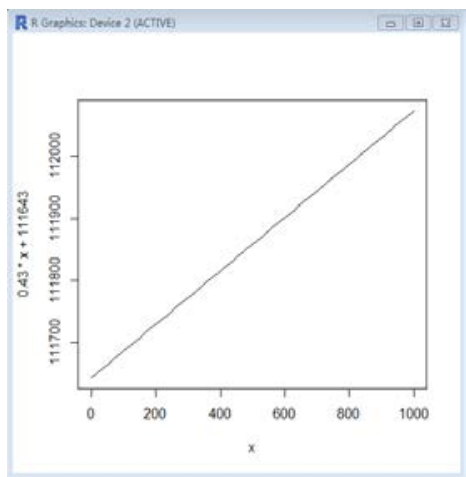

Fig4 Relationship between medium level of service quality and the optimal equilibrium price

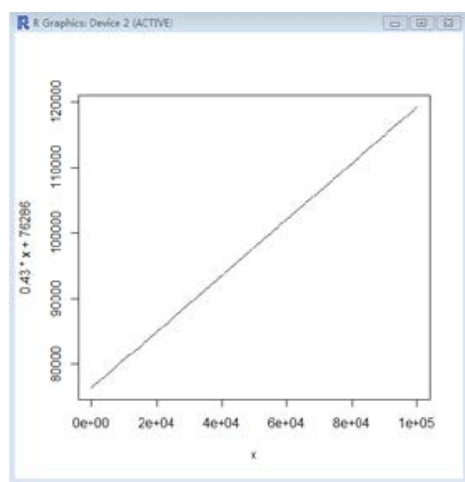

Fig5 Relationship between higher level of service quality and the optimal equilibrium price

\section{Discussion}

Though Fig 3, 4 and 5, when the competition of quality of service of 10, 1000 and 100000 respectively, the enterprise quality of service and the relationship between the optimal equilibrium price coefficient is 0.43 , with the improvement of service quality, the optimal equilibrium price also increase accordingly. Competitors' service quality does not affect the company's service quality and the relationship between the optimal equilibrium price, but the impact minimum optimal equilibrium price, the optimal equilibrium price is the lowest when the enterprise service quality equal to zero, the optimal equilibrium price at this time. When competitor's service quality is at a lower level, minimum optimal equilibrium price is highest. When a competitor's service quality improvement, the enterprise is to maximize the total profit, it must have the superior price, and the optimal equilibrium price is relatively lower.

\section{Acknowledgement}

This work is supported by National key projects of philosophy and social science fund (14ATQ006), Jiangsu Natural Science Foundation of College and University (14KJB310014) and Natural science fund of Nantong University (03041179).

\section{References}

[1]Wang Wei. Dynamic Pricing Model of Database Product Based on Lifecycle [J].Information Studies: Theory \& Application.2012, (02): 301-307.

[2] J.L. Umamaheswari. Exploring Internal Service Quality in a Manufacturing Organization - A Study in Lucus TVS, Chennai [J].Procedia Economics and Finance, 2014,11:710-725.

[3]A. Arafa, W.H. EIMaraghy. Enterprise Strategic Flexibility[J]. Procedia CIRP, 2012, 3: 537-542.

[4] Olivier Rubel. Stochastic competitive entries and dynamic pricing [J].European Journal of Operational Research.2013, 231(2):381-392. 
[5] Lata Septem Riza, Andrzej Janusz, Christoph Bergmeir. Implementing algorithms of rough set theory and fuzzy rough set theory in the R package "RoughSets"[J]. Information Science, 2014(287):68-69. 